\section{Estrategia metodológica para la elaboración y solución de problemas en química centrada en la simetría del icosaedro}

\author{
Pedronel Araque Marín ${ }^{1}$, Javier Sierra \\ Torres $^{1}$ \\ 1 Universidad EIA-Escuela de Ingeniería de Antioquía \\ \{pedronel.araque; javier.sierra\}@eia.edu.co \\ DOI https://doi.org/10.33412/pri.v9.1.2065
}

\section{(잉(อ}

Resumen: La búsqueda de un trabajo colaborativo que impacte el quehacer educativo en el aula de clase, donde se construya un lenguaje en química vital en los procesos de enseñanzaaprendizaje, especialmente para construir e interpretar situaciones problema; nos lleva al planteamiento de una estrategia metodológica centrada en la simetría del icosaedro para la elaboración y solución de problemas en química. La metodología se desarrolló en seis momentos fundamentales, basados en un ciclo didáctico, con el fin de garantizar a los estudiantes su participación como: diseñadores, críticos, rediseñadores, resolutivos y evaluadores. Durante la implementación de la estrategia se encontraron dificultades en comprensión lectora e interpretación a la hora de resolver un ejercicio, la escritura de la situación problema presentó deficiencias estructurales de forma, manejo conceptual e información inconexa. Al momento de replantear los problemas se observaron fallas en manejo de sinonimia y proformas. También se identificó y llamó profundamente la atención, la dificultad al momento de aplicar conceptos básicos de Aritmética y Álgebra. La estrategia ha potencializado en los estudiantes el reescribir, justificar, describir, definir, reflexionar y por supuesto a argumentar y contra argumentar. De esta forma lo aprendido cobra sentido y su aprendizaje es significativo y colaborativo.

Palabras clave: aprendizaje significativo, química, icosaedro, roles, competencias.

Title: Methodological strategy for the formulation and solution of problems in chemistry centered on the symmetry of the icosahedron.

\footnotetext{
Abstract: The search for a collaborative work that impacts the educational task in the classroom, where a language in vital chemistry is built in the teaching-learning processes, especially to construct and interpret problem situations; takes us to the approach of a methodological strategy centered on the symmetry of the icosahedron and based on its synergy and integrity for the development and solution of problems in chemistry. The methodology was developed in six fundamental moments, based
}

on a didactic cycle, to guarantee the participation of the students as: critical designers, re-designers resoluteness and evaluators. During the implementation of the strategy, difficulties were found in the comprehension and interpretation of the reading when solving an exercise, the writing of the problem situation presented structural deficiencies of form, conceptual handling and disconnected information. At the time of rethinking the problems, there were flaws in the use of synonymy and preformats. Also identified and deeply called attention was the difficulty at the time of applying the basic concepts of Arithmetic and Algebra. The strategy has allowed students to rewrite, justify, describe, define, reflect and, of course, argue and argue against; In this way what is learned makes sense and their learning is meaningful and collaborative.

Key words: significant learning; chemistry, icosahedron, roles, competences.

Tipo de artículo: original

Fecha de recepción: 28 de mayo de 2018

Fecha de aceptación: 17 de octubre de 2018

\section{Introducción}

Las investigaciones sobre la enseñanza de la Química se han orientado, en los últimos años, a la identificación de ideas previas en la estructura cognitiva y progreso conceptual de los estudiantes en diferentes metodologías de intervención en el aula [1]. Pero no solo los procesos de enseñanza y aprendizaje se centran en las ciencias naturales. Estos se encuentran relacionados con las ciencias sociales y ciencias del lenguaje, en contextos educativos de diversidad sociocultural, cuyas expectativas son orientar, acompañar y diseñar metodologías educativas que puedan responder a la necesidad de una región y fortalecer una red de investigación en educación [2].

Es evidente que en las clases de ciencias los estudiantes tienen que aprender los modelos científicos y los términos especializados que forman parte de estos modelos, pero también deben empezar a hablar de los fenómenos con sus propias palabras [3], y estas irán cambiando a medida que adquieran nuevos conceptos. De alguna manera, la actividad científica, también es una actividad lingüística. [4].

Entre los procesos de enseñanza-aprendizaje de la química es importante realizar tres reflexiones: la primera consiste en conocer cómo tiene estructurado el estudiante los conocimientos del tema, para orientar la construcción de conceptos adecuados, con el fin de correlacionar su saber existente en la estructura cognitiva con los nuevos conocimientos aportados por las actividades propuestas en el aula de clase.

La segunda consiste en proporcionar un verdadero aprendizaje significativo porque desconocerlo nos induce a entregar información inadecuada a los estudiantes suponiendo que "lo deben saber", pero solo están copiando la información para memorizarla, reproducirla y finalmente olvidarla, y la tercera tiene que ver con el proceso de evaluación formativa que facilite la valoración del desempeño de los estudiantes incrementando la 
objetividad de un área que ha sido considerada subjetiva y compleja.

El desarrollo de prácticas evaluativas justas y que sean además transparentes, siguiendo principios democráticos (participativos e incluyentes); estos principios se volverían indispensables en los lineamientos a seguir en el diseño de procedimientos de un sistema de evaluación que garantice un impacto positivo en los procesos de enseñanza-aprendizaje, el cual se puede traducir en una evaluación para el aprendizaje y debe ser el principal objetivo que guíe todo evento evaluativo en el aula [5], esta metamorfosis trae modificaciones en el proceso de evaluación, considerando que la evaluación no puede limitarse a un solo tipo de prueba centrada en la repetición de contenidos y que pretenda solo alcanzar una calificación [6].

En el tema de evaluación, considerado como parte fundamental en el proceso de enseñanza-aprendizaje, se ha propuesto un conjunto de características de una prueba constituida por tareas llamada rúbrica [7]. Además, vista como un instrumento de evaluación [8], se basa en una escala cualitativa y/o cuantitativa asociada a criterios que puedan medir las acciones de los estudiantes sobre los aspectos de las actividades que serán evaluadas [9] y desde estos criterios, poder juzgar, valorar, calificar y conceptualizar sobre un determinado aspecto del proceso, existiendo una conexión entre la enseñanza y la evaluación, es decir debe existir una concordancia entre lo que se está enseñando y lo que se está midiendo [10]. La escala holística presenta una descripción general de los diferentes niveles de logro esperado en el desempeño de los estudiantes respecto a una tarea determinada.

La escala analítica describe cada uno de los elementos del marco definido como constructo para cada nivel de logro de la escala, siendo estas más efectivas en procesos de evaluación en el aula dado que posibilita una realimentación detallada, descriptiva y dialógica entre el profesor y el estudiante [11].

Entre los trabajos colaborativos que impacten el quehacer educativo en el aula, y en especial la utilización de herramientas metodológicas no convencionales, se encuentra el juego de roles, el cual es entendido como "técnica útil para manejar aspectos 0 temas difíciles en los que es necesario tomar diferentes posiciones para su mejor comprensión" [12] y el método syntegrity [13], conocido como el método del icosaedro, basado en las características de la sinergia e integridad de la estructura de un poliedro regular como el icosaedro por sus propiedades de simetría lo hacen libre de desigualdades, con información distribuida dentro de sus 12 vértices, 20 caras y 30 aristas, siendo las 30 aristas lo correspondiente al número de estudiantes alrededor de los cuales se diseñará la estrategia para resolver una situación problema (figura 1).

Con el ánimo de solventar la necesidad encontrada en cuanto a las dificultades de comprensión lectora e interpretación, aspectos fundamentales a la hora de elaborar y resolver un ejercicio de aplicación en química, se propone una estrategia metodológica que permita mostrar, no solo su implementación sino su aplicación como coadyuvantes de la potenciación de las

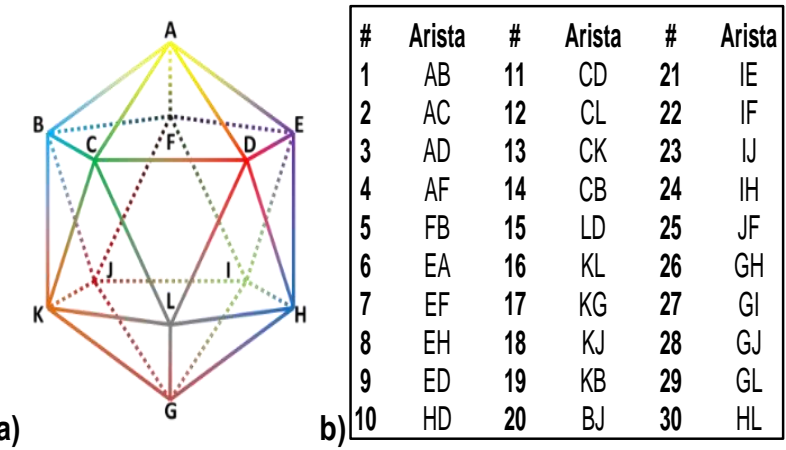

Figura 1. Distribución de los estudiantes en el aula de clase como las aristas del icosaedro.

competencias, comunicativa, de trabajo en equipo, de pensamiento sistémico y de creatividad.

\section{Metodología}

Con el propósito de implementar una estrategia metodológica para la elaboración y solución de problemas en química centrada en la simetría del icosaedro, en la asignatura de Química General e Inorgánica, para estudiantes de primer semestre de ingeniería en la Universidad EIA, durante los periodos académicos 2017-1 y 2017-2. La distribución por género fue de $58 \%$ hombres y $42 \%$ mujeres.

Inicialmente se implementó el método syntegrity, como método comparativo, el cual consistió en dividir el grupo de 30 estudiantes en dos subgrupos de 15 estudiantes cada uno, donde tres subgrupos de cinco estudiantes, asumirán los roles de diseñadores, críticos, resolutivos y evaluadores.

Posteriormente, la estrategia centrada en la simetría del icosaedro, se realizó en cinco sesiones con un grupo de 30 estudiantes. En la primera sesión se dividió el grupo en seis subgrupos de cinco estudiantes y a cada subgrupo se asignó el rol de diseñadores. En la segunda sesión se dividió el grupo en seis subgrupos y se asignó el rol de críticos. En la tercera sesión se retomó la agrupación de los diseñadores, pero ya con el rol de rediseñadores y la cuarta sesión se retomó la agrupación de los críticos, pero ya con el rol de resolutivos y la quinta sesión, se dividió el grupo en seis subgrupos y se asignó el rol de evaluadores.

La metodología se desarrolló en seis momentos fundamentales, basados en un ciclo didáctico [14], como se describen a continuación:

\subsection{Elaboración de la situación problema}

Se asignó una sustancia a cada subgrupo de estudiantes, para que asumieran el rol de diseñadores y elaboraran de modo escrito un problema de estequiometria, donde la sustancia (representada por la estructura química) se tiene que dilucidar a partir de reacciones químicas y plantear la posible fórmula molecular. 


\subsection{Comprensión e interpretación del problema}

Se realizó iteración de los participantes construyendo nuevos subgrupos a los que se les asignó el rol de críticos. Su papel fue emitir argumentos de oposición desde la comprensión lectora de la propuesta de los diseñadores. Además de generar una oposición respetuosa de la escritura, plantearon una posible solución al problema.

\subsection{Replanteamiento del enunciado del problema}

Se realimentó el grupo de los diseñadores con las observaciones realizadas por los críticos desde la comprensión lectora del ejercicio hasta las dificultades para dar solución al mismo, por falta de algunos datos dentro del enunciado. De esta forma los diseñadores, asumiendo el papel de rediseñadores construyeron un nuevo enunciado de la situación problema.

\subsection{Solución de la situación problema}

A partir de las correcciones realizadas por los diseñadores, los críticos, asumiendo el papel de resolutivos, propusieron una solución a la situación problema.

\subsection{Implementación del instrumento de evaluación}

Se asignó a los estudiantes el rol de evaluadores. Su papel, por medio de la rúbrica como instrumento de evaluación, basado en una escala cuantitativa y asociada a criterios, les permitió medir la construcción de la situación problema de los diseñadores y las soluciones planteadas por los críticos.

\subsection{Autoevaluación}

Teniendo en cuenta los resultados de las evaluaciones, los mismos evaluadores se reunieron para interpretar desde la implementación de la rúbrica como instrumento de autoevaluación y de esta forma construir un proceso de realimentación que les permita conocer sus fortalezas y debilidades.

\section{Resultados}

\subsection{Estrategia basada en el método syntegrity}

El método syntegrity, está basado en las características de la sinergia e integridad de la estructura del icosaedro, por sus propiedades simétricas que lo hacen libre de desigualdades, con información distribuida dentro de sus 12 vértices, 20 caras y 30 aristas, siendo las 30 aristas el número de estudiantes alrededor de una situación problema. La metodología consiste en simular que las 30 aristas del icosaedro corresponden a dos grupos de 15 estudiantes, donde tres subgrupos de cinco estudiantes asumirán los roles de diseñadores (pirámide pentagonal), críticos (pentágono opuesto a la pirámide pentagonal) y evaluadores (arista de enlace entre la base piramidal y el pentágono). En la figura 2 se representan las diferentes posibilidades de cómo se pueden organizar los estudiantes distribuyendo lo roles mencionados.

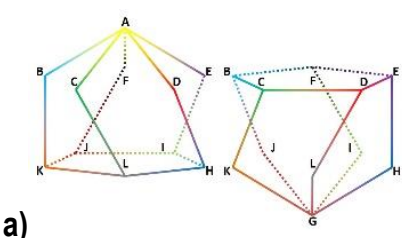

a)

c)

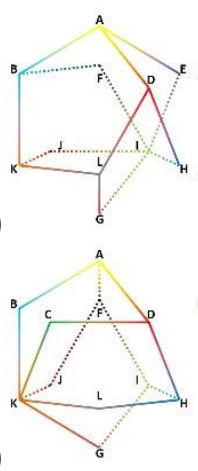

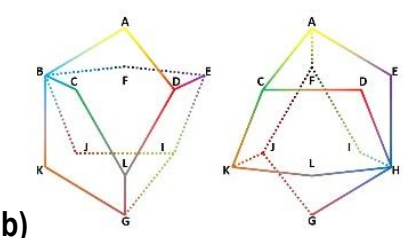

b)

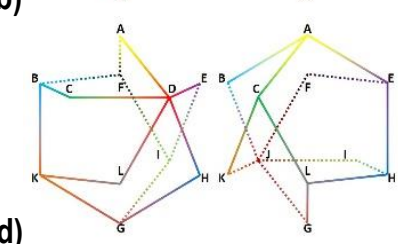

d)
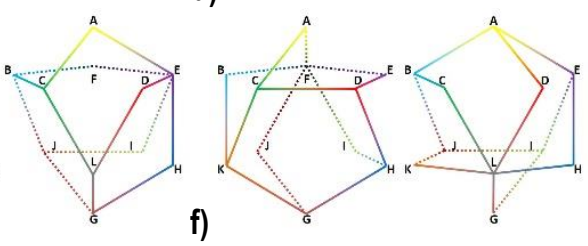

Figura 2. Representación de las posibilidades del método syntegrity: a) Icosaedro 1; b) Icosaedro 2; c) Icosaedro 3; d) Icosaedro 4, e) Icosaedro 5; f) Icosaedro 6.

Las seis posibilidades que se representan en la figura 2 , son necesarias para que cada estudiante tenga como mínimo participación en los roles de diseñador, crítico y evaluador. Un ejemplo de esto es el caso del estudiante 2 representado como la arista $\mathrm{AC}$ en las figuras 3 y 4 .

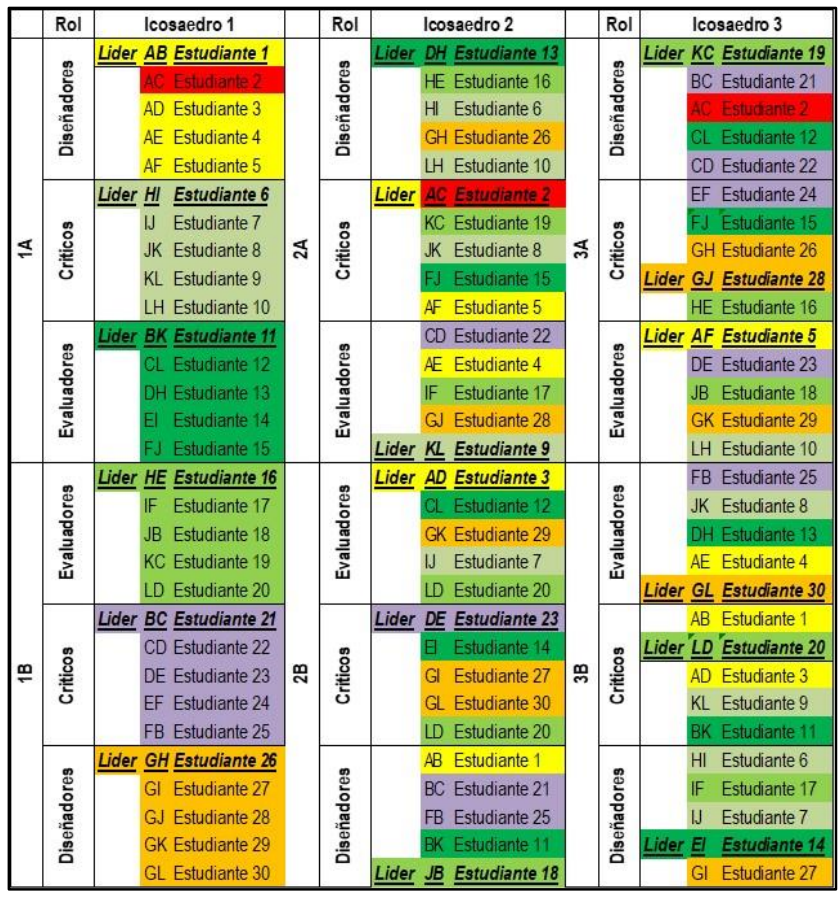

Figura 3. Organización de los estudiantes basados en el método syntegrity para los icosaedros 1,2 y 3 . 
La implementación de esta metodología en la primera sesión presentó dificultades, dado que el grupo de estudiantes diseñadores se tomaron 45 minutos para la construcción de la situación problema. Durante este tiempo los críticos y los evaluadores que participaban como observadores se dispersaron, concentrándose en otras actividades diferente a la planteada en clase. Posteriormente los críticos se tomaron 30 minutos para generar oposición respetuosa a los argumentos. Igualmente, durante este tiempo los diseñadores y los evaluadores se dispersaron durante la actividad.

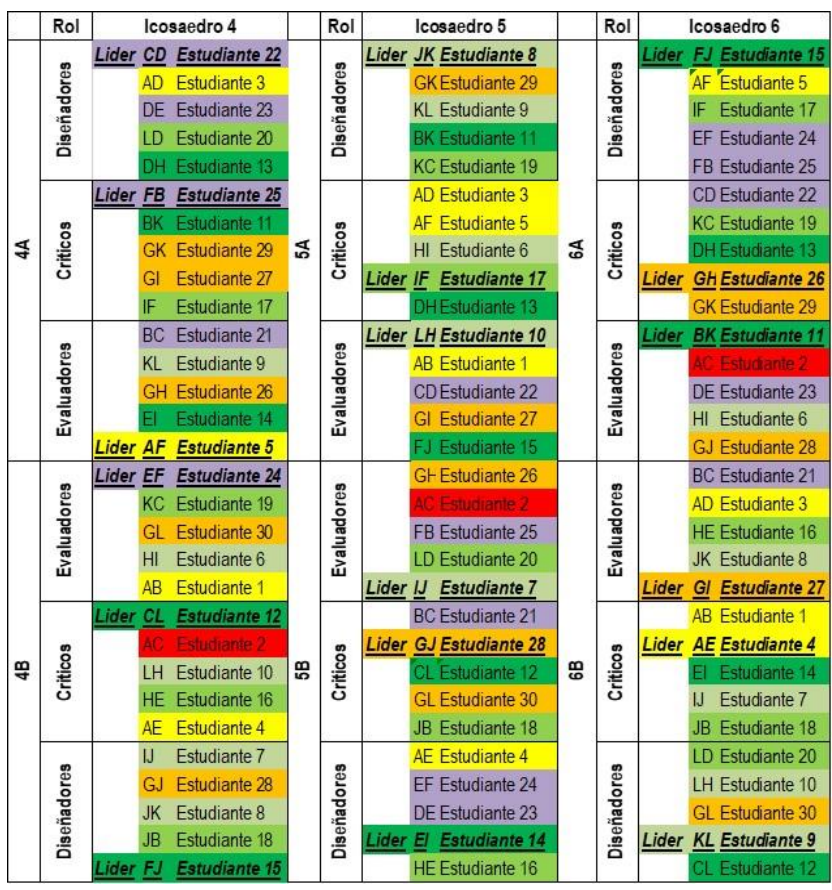

Figura 4. Organización de los estudiantes basados en el método syntegrity para los icosaedros 4,5 y 6 .

Los estudiantes con los roles de críticos y evaluadores manifestaron su inconformidad, dado que querían también ser diseñadores. Sin embargo, fueron los evaluadores quienes estuvieron más desmotivados. En primer lugar, por el tiempo que tuvieron que esperar y en segundo lugar porque la extensión de los diseñadores y críticos no daba tiempo para su participación, ya que la clase había terminado. Por lo anterior, se planteó una modificación al método syntegrity con el propósito de que cada estudiante del grupo tomara el rol de diseñador, crítico y evaluador, además asignaran roles dentro de los subgrupos y de esta forma garantizar un trabajo colectivo.

\subsection{Estrategia centrada en la simetría del icosaedro}

La modificación al método syntegrity, consistió en construir a partir de los 30 estudiantes seis subgrupos donde se garantizará que todos los estudiantes pudieran tener el rol de diseñadores, críticos y evaluadores, en un mínimo número de sesiones sin perder la motivación. Además que la estrategia permita mostrar, no solo su implementación sino su aplicación como coadyuvantes de la potenciación de las competencias: comunicativa, de trabajo en equipo, de pensamiento sistémico y de creatividad.

\subsubsection{Planteamiento de la situación problema}

En la figura 5 se observan los subgrupos de estudiantes diseñadores como parte del icosaedro. Los subgrupos designaron internamente roles resaltando las habilidades y aptitudes de cada integrante. En la figura 6, se presentan las sustancias químicas asignadas a cada subgrupo de diseñadores y a partir de ella elaboraran de modo escrito un problema de estequiometria, donde la sustancia (representada por la estructura química) se tiene debe construir a partir de reacciones químicas y de esta forma proponer una posible formula molecular. a)

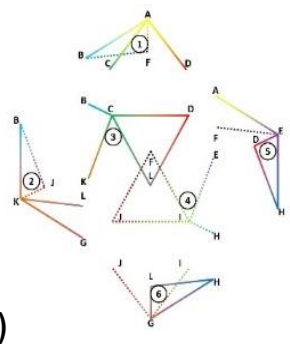

b)

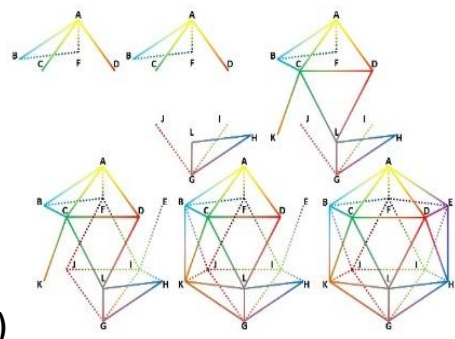

Figure 5. Representación de los diseñadores: a) subgrupo de estudiantes; b) icosaedro como aula de clase.

\section{(1) Ibuprofeno $\mathrm{C}_{13} \mathrm{H}_{18} \mathrm{O}_{2}$ (4) Triptoffano $\mathrm{C}_{11} \mathrm{H}_{12} \mathrm{~N}_{2} \mathrm{O}_{2}$ \\ (2) Cafeina $\mathrm{C}_{8} \mathrm{H}_{10} \mathrm{~N}_{4} \mathrm{O}_{2}$ (5) Nicotina $\mathrm{C}_{10} \mathrm{H}_{14} \mathrm{~N}_{2}$ \\ (3) Metionina $\mathrm{C}_{5} \mathrm{H}_{11} \mathrm{NO}_{2} \mathrm{~S}$ (6) Endosulfán $\mathrm{C}_{9} \mathrm{H}_{6} \mathrm{Cl}_{6} \mathrm{O}_{3} \mathrm{~S}$}

Figure 6. Sustancias asignadas y su respectiva fórmula molecular.

En las situaciones problemas propuestas se evidenciaron problemas de sintaxis y ortografía que afectan la cohesión y coherencia, indispensables interpretación y comprensión del problema y la elaboración del enunciado. Se presentan deficiencias en el manejo de verbos y en la cohesión del planteamiento del problema; además no tienen en cuenta el correcto uso de mayúsculas, signos de puntuación y formulación de preguntas. Los estudiantes conceptualizaron adecuadamente el proceso de proponer una fórmula molecular, ya que se evidencia que todos los cálculos estequiométricos fueron planteados para que cumplieran tal fin. Después se presentaron dudas al momento de escribir algunas fórmulas moleculares y dificultades para crear un contexto donde se pudiera integrar la sustancia problema. Algunos grupos no diferenciaron ciertos elementos químicos y sus respectivas masas molares, lo que llevó a confundir elementos dentro de una fórmula molecular. 


\subsubsection{Comprensión e interpretación de la situación problema}

En la figura 7 se observan los subgrupos de estudiantes críticos como partes del icosaedro. Los subgrupos fueron construidos de tal forma que el ejercicio asignado para emitir argumentos de oposición desde la comprensión lectora, fue diferente al que formularon cuando fueron diseñadores. En el informe presentado por los críticos se evidenciaron problemas de sintaxis y ortografía que afectan la cohesión y coherencia; no se completan algunas palabras, utilizando abreviaturas inadecuadas, se presentó déficit en el manejo de signos de interrogación y en la cohesión del planteamiento de las preguntas; además no tienen en cuenta el uso correcto de mayúsculas y signos de puntuación.

Partiendo de los argumentos presentados por los críticos sobre la dificultad en comprensión lectora del ejercicio planteado por los diseñadores, se encontró que la escritura de la situación problema presentaba dificultades estructurales de forma, manejo conceptual en química y manejo de información inconexa, debido a la no contextualización de la situación problema.

Los estudiantes presentaron deficiencias conceptuales entre la fórmula mínima y molecular, en las relaciones estequiometrias y la ley de la conservación de la masa.

a)

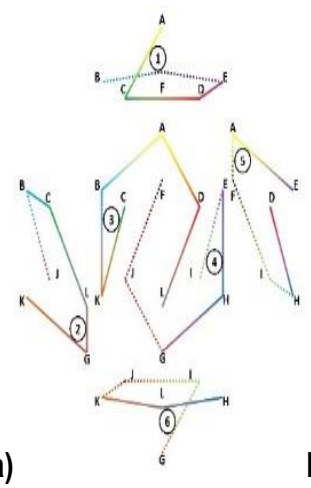

b)

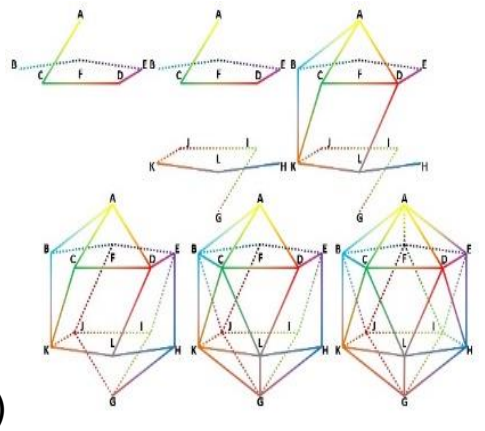

Figure 7. Representación de los críticos: a) subgrupo de estudiantes; b) icosaedro como aula de clase.

\subsubsection{Replanteamiento del enunciado de la situación problema}

Teniendo en cuenta las recomendaciones de los críticos, los diseñadores replantearon la situación problema, pero en sus propuestas se presentaron nuevamente errores en la estructura formal, problemas de sintaxis y ortografía. Los diseñadores se organizaron en dos subgrupos (figura 8) y se compartieron las dificultades de comprensión e interpretación desde la cohesión y coherencia de los enunciados, considerados como los elementos indispensables para la comprensión del problema y la elaboración del enunciado.

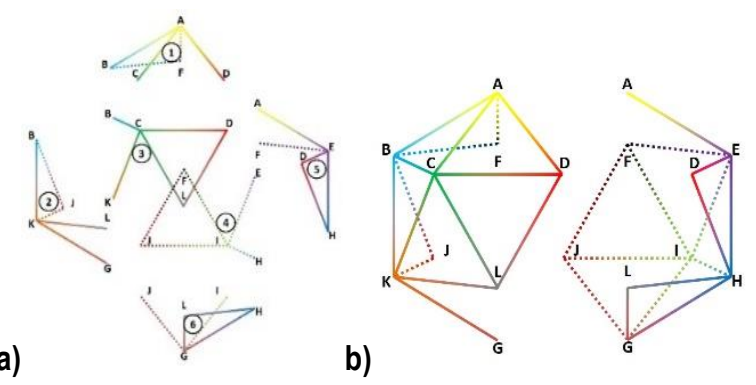

Figure 8. Representación de los rediseñadores: a) subgrupo de estudiantes; b) icosaedro como aula de clase.

Finalmente, superada la conceptualización y contextualización, los diseñadores propusieron sus enunciados. A continuación se comparten algunos de ellos:

1. Un deportista duda si el contenido del recipiente es ibuprofeno $\left(\mathrm{C}_{13} \mathrm{H}_{18} \mathrm{O}_{2}\right)$ o buprenorfina $\left(\mathrm{C}_{29} \mathrm{H}_{41} \mathrm{NO}_{4}\right)$ esta última considerada sustancia prohibida por la organización antidopaje. Antes de consumirla el equipo médico envía a un laboratorio una muestra de $4.677 \mathrm{~g}$ del analgésico, el cual es sometido a combustión completa obteniendo $3.690 \mathrm{~g}$ de $\mathrm{H}_{2} \mathrm{O}_{\text {() }}$ y $7.194 \mathrm{~L}$ de $\mathrm{CO}_{2(\mathrm{~g})}$ medido a $750 \mathrm{mmHg}$ y $25^{\circ} \mathrm{C}$. En un segundo laboratorio analizaron una muestra de $9.354 \mathrm{~g}$ la cual reducen

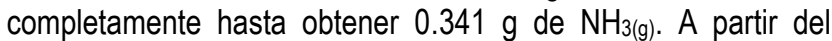
proceso realizado ¿puede el deportista tomar el medicamento para continuar en competencia?

2. Un científico asegura que inventó un proceso para transformar cafeína $\left(\mathrm{C}_{8} \mathrm{H}_{10} \mathrm{~N}_{4} \mathrm{O}_{2}\right)$ en teobromina $\mathrm{C}_{7} \mathrm{H}_{8} \mathrm{~N}_{4} \mathrm{O}_{2}$. Con el fin de verificar el proceso, algunos colegas someten el producto a oxidación completa poniendo en reacción la sustancia con óxido cúprico $\mathrm{CuO}_{(\mathrm{s})}$. De la oxidación de $0.400 \mathrm{~g}$ de muestra, los vapores de agua $\mathrm{H}_{2} \mathrm{O}_{(\mathrm{g})}$ se hacen pasar sobre una muestra de perclorato de magnesio, la cual aumenta en $0.160 \mathrm{~g}$ su masa, los vapores que se producen de $\mathrm{CO}_{2(\mathrm{~g})}$ ocupan un volumen de 380 $\mathrm{mL}$ a $25{ }^{\circ} \mathrm{C}$ y $1 \mathrm{~atm}$. Posteriormente, tomaron una segunda muestra de $0.200 \mathrm{~g}$ y de la combustión completa obtuvo $0.109 \mathrm{~L}$ de $\mathrm{NO}_{2(\mathrm{~g})}$ a $23{ }^{\circ} \mathrm{C}$ y $750 \mathrm{mmHg}$. A partir del proceso utilizado, determinar si la cafeína se transformó en teobromina.

3. Para acelerar la maduración de frutos se recomienda aplicar un aminoácido azufrado como metionina $\left(\mathrm{C}_{5} \mathrm{H}_{11} \mathrm{O}_{2} \mathrm{NS}\right)$. Sin embargo, el cultivo se marchitó rápidamente. El marchitamiento se da por uso de cisteína $\left(\mathrm{C}_{3} \mathrm{H}_{7} \mathrm{O}_{2} \mathrm{NS}\right)$ que es otro aminoácido azufrado, o por la aplicación inadecuada de la metionina. Un ingeniero tomó una muestra de $4.380 \mathrm{~g}$ y la sometió a combustión completa produciendo: $2.279 \mathrm{~g} \mathrm{de} \mathrm{H}_{2} \mathrm{O}_{(\mathrm{l})}, 0.964 \mathrm{~L}_{\text {de }} \mathrm{SO}_{2(\mathrm{~g})}$ y 2.887 $\mathrm{L}$ de $\mathrm{CO}_{2(\mathrm{~g})}$ a $24.5^{\circ} \mathrm{C}$ y $698 \mathrm{mmHg}$; luego para determinar el contenido de nitrógeno, tomó una segunda muestra de $2.190 \mathrm{~g}$ y la sometió al método Kjedahl obteniendo una cantidad de $\mathrm{NH}_{3(g)}$ que se disolvió en $500 \mathrm{~mL}$ de agua produciendo una solución

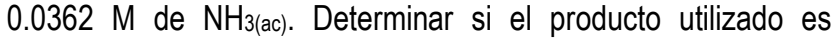
metionina. 


\subsubsection{Solución de la situación problema}

A partir del problema replanteado por los diseñadores, los críticos se reúnen y toman el rol de resolutivos y de esta forma proponen una reestructuración del trabajo en el aula (figura 9), y compartir material necesario para la solución de los problemas como: tabla periódica, calculadoras, tablas de constantes, computadores entre otros.

a)

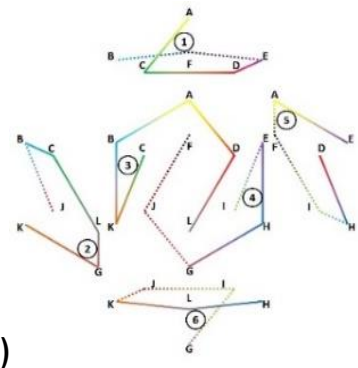

b)

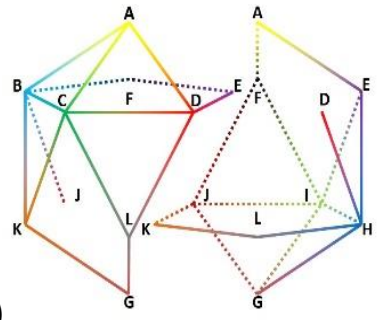

Figure 9. Representación de los resolutivos: a) subgrupo de estudiantes; b) icosaedro como aula de clase.

\subsubsection{Evaluación de la solución de la situación problema}

En la figura 10 se observan los subgrupos de estudiantes evaluadores como partes del icosaedro. Los subgrupos evaluadores fueron construidos de tal forma que el ejercicio fijado para otorgar la nota fue diferente al que se asignó cuando plantearon la situación problema y cuando lo cuestionaron. a)

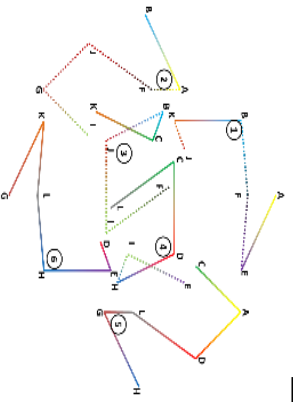

b)

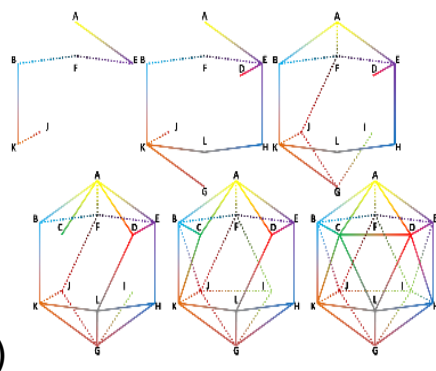

Figure 10. Representación de los evaluadores: a) subgrupo de estudiantes; b) icosaedro como aula de clase.

Los evaluadores por medio de la rúbrica (figura 11) y con el fin de evaluar el criterio "Determina la fórmula molecular de una sustancia problema que es sometida a descomposición", identificaron que las dificultades al momento de dar solución a una situación problema, no están solo en la conceptualización química o en la comprensión lectora, sino en la aplicación de conceptos básicos de Aritmética y Álgebra. Entre ellas se encuentran: cifras significativas y notación científica (uso inadecuado del resultado que entrega la calculadora y no utilizan potencias de 10); conversión de unidades (no hay claridad en el cambio de unidades y cuando deben multiplicar dividen, o lo contrario); despeje de una variable (se presenta dificultad al resolver una ecuación con una variable); casos de factorización (confusión al

momento de aplicar un determinado caso de factorización); manejo inadecuado de los signos y símbolos de agrupación (no se respeta la jerarquía que existe entre los símbolos de agrupación) y uso de la propiedad distributiva (la aplican pensando que es del producto con respecto al producto y no del producto con respecto a la suma, que es la forma correcta).

\begin{tabular}{|c|c|c|c|c|c|}
\hline \multirow[b]{2}{*}{ Dimensiones } & \multicolumn{5}{|c|}{ Niveles de desempeño } \\
\hline & $0 \%$ & $25 \%$ & $50 \%$ & $75 \%$ & $100 \%$ \\
\hline $\begin{array}{l}\text { Identfica la la } \\
\text { sustancias } \\
\text { compuestas: } \mathrm{CO}_{2} \\
\mathrm{H}_{2} \mathrm{O} \text { y } \mathrm{NH}_{3}, \mathrm{Como}_{2} \\
\text { fuente de } \\
\text { Carbono, } \\
\text { Hidrogeno y } \\
\text { Nitrógeno } \\
\text { respectivamente. }\end{array}$ & $\begin{array}{l}\text { No desarrolla } \\
\text { ningún cálculo } \\
\text { ni análisis. }\end{array}$ & $\begin{array}{l}\text { No demuestra caridad } \\
\text { en la determinación de } \\
\text { las moles de } \mathrm{CO}_{2} \mathrm{H}_{2} \mathrm{O} \text {, } \\
\mathrm{NH}_{3} \text { Muestra } \\
\text { relaciones molares } \\
\text { equivocadas entre el C } \\
\text { ye el CO entre el } \mathrm{H} \text { y el } \\
\mathrm{H}_{2} \mathrm{O} \text { y el N y el } \mathrm{NH}_{3} \text {. }\end{array}$ & $\begin{array}{l}\text { Establece relaciones } \\
\text { estequiométricas } \\
\text { adecuadamente, pero con } \\
\text { algunas imprecisiones en } \\
\text { la determinación de las } \\
\text { masas de los elementos y } \\
\text { el uso de la ecuación que } \\
\text { rige el comportamiento de } \\
\text { gases ideales al momento } \\
\text { de calculat las moles de } \\
\mathrm{CO}_{2}\end{array}$ & $\begin{array}{l}\text { Establece relaciones } \\
\text { estequiométicas } \\
\text { adecuadamente. }\end{array}$ & $\begin{array}{l}\text { Demuestra el dominio } \\
\text { de las relaciones } \\
\text { estequiométricas, para } \\
\text { determinar las moles y } \\
\text { la masas de los } \\
\text { elementos } \mathrm{C}, \mathrm{Hy} \mathrm{N} \text {. }\end{array}$ \\
\hline $\begin{array}{l}\text { Aplica la Ley de } \\
\text { conservación de } \\
\text { la massa }\end{array}$ & $\begin{array}{l}\text { No desarrolla } \\
\text { ninguńc ćliculo } \\
\text { ni anáilisis. }\end{array}$ & $\begin{array}{l}\text { No tene claridad en el } \\
\text { proceso } \\
\text { extrapolación para } \\
\text { determinar la cantidad } \\
\text { de un elemento en } \\
\text { difierentes muestras y } \\
\text { no aplica la ley de la } \\
\text { conservación de la } \\
\text { masa }\end{array}$ & 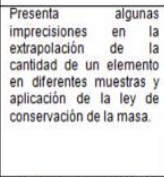 & 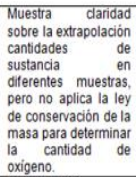 & $\begin{array}{l}\text { Demuestra dominio en } \\
\text { la extrapolación } \\
\text { cantidades de sustancia } \\
\text { en diferentes muestras } \\
\text { y plantea de modo } \\
\text { adecuado la ley de } \\
\text { conservación de la } \\
\text { masa. }\end{array}$ \\
\hline $\begin{array}{l}\text { Determina ia } \\
\text { formula minima } \\
\text { ylo molecular }\end{array}$ & $\begin{array}{l}\text { No desarrolla } \\
\text { ningúnćálculo } \\
\text { ni análisis. }\end{array}$ & $\begin{array}{l}\text { No identificalas } \\
\text { relaciones } \\
\text { estequiométricas, entre } \\
\text { elementon para la } \\
\text { determinación de la } \\
\text { fórmula molecular. }\end{array}$ & $\begin{array}{l}\text { Identifica parcialmente las } \\
\text { relaciones } \\
\text { estequiométricas, entre } \\
\text { elementos, pero no tiene } \\
\text { daridad sobre el pla } \\
\text { procedimiento para } \\
\text { determinar la tormula } \\
\text { molecular. }\end{array}$ & $\begin{array}{l}\text { Identifica las } \\
\text { relaciones } \\
\text { estequiométricas, } \\
\text { entre elementos, pero } \\
\text { no tiene claridad } \\
\text { sobre el } \\
\text { procedimiento para } \\
\text { determinarla formula } \\
\text { molecular. }\end{array}$ & $\begin{array}{l}\text { Demuestra el dominio } \\
\text { de las relaciones } \\
\text { estequiométricas, y } \\
\text { tiene daridad sobre el } \\
\text { procedimiento para } \\
\text { determinar la tormula } \\
\text { molecular. }\end{array}$ \\
\hline
\end{tabular}

Figure 11. Rúbrica para la evaluación del criterio: "determina la fórmula molecular de una sustancia problema" que es sometida a descomposición.

El diseño de la rúbrica logró establecer características claves para el proceso de implementación como la determinación de los factores que indicarán la calidad del trabajo de los estudiantes en términos de objetivos de aprendizaje y criterios de evaluación, proporcionando una explicación detallada de lo que el estudiante debe realizar para demostrar sus niveles de eficiencia y de esta forma alcanzar los niveles determinados en los objetivos e impulsando la construcción de un sistema de evaluaciones formativas más coherente con las actuaciones de los profesores y estudiantes.

\subsubsection{Autoevaluación de la solución de la situación problema}

Finalmente, los evaluadores se reagruparon (figura 12) para compartir los resultados y proceder a una autoevaluación.

a)

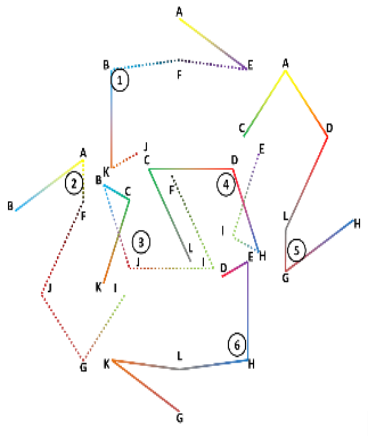

b)

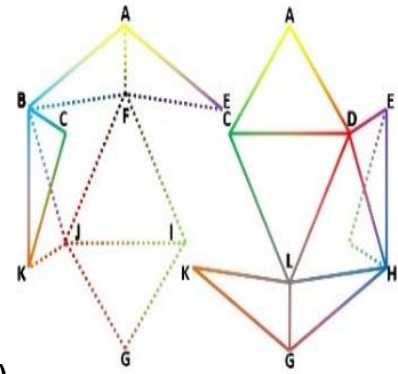

Figure 12. Representación de los autoevaluadores: a) subgrupo de estudiantes; b) icosaedro como aula de clase. 
El análisis comparativo dentro de cada subgrupo y el proceso de realimentación de la rúbrica les permitió conocer más sobre su fortaleza y debilidad en el proceso de aprendizaje. Además, manifestaron que las rúbricas fueron valiosas no solo como elemento de evaluación sino como instrumento para la reflexión y la comunicación.

Aunque la autoevaluación no es una práctica habitual y el desconocimiento de su utilidad pedagógica en el aula de clase ha fomentado una fragmentación en el proceso evaluativo, nos lleva a reflexionar sobre la importancia de la construcción de materiales potencialmente significativos donde se suministre información precisa acerca de los aspectos y/o indicadores que se consideran en una evaluación y de esta forma el estudiante pueda evaluar continuamente su propio aprendizaje, así como registrar e interpretar objetivamente la información que el proceso evaluativo suministra, y de esta forma puedan tomar decisiones oportunas que garanticen el mejoramiento en su proceso formativo.

En la figura 13 se esquematiza la organización del grupo de estudiantes en los cinco roles: diseñadores, críticos, rediseñadores, resolutivos y evaluadores. Además, se puede observar los seis subgrupos en cada rol, en cada subgrupo se tiene un estudiante líder quien es el responsable de delegar y coordinar las funciones dentro del equipo de trabajo.

Es importante resaltar que los estudiantes diseñadores no criticarán ni evaluarán la situación problema que construyeron, como lo indican los colores. En la figura 13 se resalta en color rojo el estudiante seis representado como la arista AC del icosaedro, para mostrar su trazabilidad durante la implementación de la estrategia.

\begin{tabular}{|c|c|c|c|c|c|}
\hline Subgrupo & Diseñadores & Criticos & Rediseñadores & Resolutivos & Evaluadores \\
\hline & Lider AB Estudiante 1 & Lider GI Estudiante 2 & AB Estudiante 1 & GI Estudiante2 & r LC Estudiante \\
\hline & & IJ Estudiante 28 & & IJ Estudiante 28 & CD Estudiante 2 \\
\hline & AD Estudiante 11 & JK Estudiante 20 & Lider AD Estudiante 11 & JK Estu & DH Est \\
\hline & $A F E S$ & KL Estu & $A F E S$ & KL. E & \\
\hline & $\mathrm{FB} E S$ & HL. Est & & er $\underline{H L}$ & \\
\hline \multirow{5}{*}{2} & Lider GH Estudiante 26 & Estudiante 6 & GH Es & & \\
\hline & GI Estu & CD Estudiante 22 & $\mathrm{Gl} \mathrm{E}$ & CD Estu & CBE: \\
\hline & GJ Es & DE Estudi & Lider GJ Es & DE Estu & BJ E: \\
\hline & GL Es & EF Estuc & & & ider $\| \underline{E}$ \\
\hline & HL Es & FB Estur & HL E & Lider FB & \\
\hline \multirow{5}{*}{3} & $\operatorname{der} \underline{C D} \underline{E s t}$ & IE Estl & & & Lider JK \\
\hline & CL. Estu & EH Estuc & & Lider 태 Ess & $\overline{\mathrm{KB}} \mathrm{E}$ \\
\hline & Est & HG Estuc & CK Estu & HG Est & $\mathrm{BF} E$ \\
\hline & Est & GJ Est & $C B$ E & & \\
\hline & 13 & Lider JF Est & & JF Es & \\
\hline \multirow{5}{*}{ L } & Lider IE Est & SLider CK Estu & $\mathbb{E E}$ & CK Estt & GKE \\
\hline & IF Estud & KB Estud & Lider IF Estud & KB Est & \\
\hline & IJ Estudiante 28 & BA Estur & if Estudiante 28 & BA Es & LHE \\
\hline & $\mathbb{1 H}$ Estudi & AD Estur & IH Estud & AD Estu & HEE \\
\hline & JF Estud & DL Estu & JF Estu & Lider DL Estudiante 13 & Lider EDE \\
\hline \multirow{5}{*}{5} & Lider EA Estudiante 14 & 4Lider KG Estudiante 15 & EA Est & $\overline{K G}$ Est & $\overrightarrow{B A} \bar{E}$ \\
\hline & EF Estudiante 19 & GL. Estudiante 12 & Lider EF Estu & GL Est & Lider AFE \\
\hline & EH Estu & LC Estud & EH Estu & LC Esti & \\
\hline & & CR Fotur & ED E & & \\
\hline & HD E & BJ Esth & $\mathrm{HDE}$ & Lider $B J$ & \\
\hline \multirow{5}{*}{6} & Lider $\underline{K L} \underline{E s t}$ & EA Estud & KL ES & & \\
\hline & KG Estuc & AF Estudiante 16 & KG Estu & AF Estudiante 16 & AD Estudiante 1 \\
\hline & KJ Estuc & $\mathrm{Fl}$ Estudiante 23 & $\mathrm{KJ}$ Estudiante 20 & Fl Estudiante 23 & DL Estudiante \\
\hline & KB Estu & IH Estudi & Lider KB Estudiante 25 & Lider IH Estudiante 4 & Lider $\underline{L G}$ Estud \\
\hline & BJ Estudiante 30 & Lider $\mathrm{HD}$ Estudiante 5 & BJ Estudiante 30 & HD Estudiante 5 & GH Estudiante? \\
\hline
\end{tabular}

Figure 13. Estrategia metodológica organizada en los subgrupos: diseñadores; críticos y evaluadores.

\section{Conclusión}

Con la estrategia metodológica los estudiantes fueron partícipes de la creación de una situación problema, dieron solución a dos ejercicios y evaluaron uno. Durante la actividad, además todos los estudiantes tuvieron el papel de líder, el cual fue de gran significado para el trabajo en equipo.

La estrategia ha potencializado en los estudiantes el reescribir, justificar, describir, definir, reflexionar y por supuesto a argumentar y contra argumentar; lo que hace que lo aprendido cobre sentido para ellos y que en últimas su aprendizaje sea significativo y colaborativo.

La utilización de esta herramienta metodológica no convencional promueve el desarrollo y evolución de las competencias: de creatividad, de pensamiento sistémico, trabajo en equipo y comunicativa. Se pudo detectar que las dificultades al momento de dar solución a una situación problema no estaban en solo en la comprensión lectora, sino en conceptos básicos de la aritmética.

Los diseñadores rescataron la importancia de la competencia comunicativa al momento de diseñar una situación problema, rescatando que lo importante en la construcción de un ejercicio es hacerlo de tal forma que otra persona lo pueda leer y entender.

Los estudiantes manifestaron que el proceso de realimentación a través de la rúbrica les permitió conocer más sobre sus fortalezas y debilidades en el proceso de aprendizaje. También, destacan que las rúbricas, además de ser valiosas como elemento de evaluación, son, nuevamente, instrumentos para la reflexión y la comunicación.

La implementación de la rúbrica como instrumento de autoevaluación llama a la reflexión sobre la adaptación al contexto curricular, ya que los estudiantes manifestaron que el proceso de realimentación les permitió conocer sus fortalezas y debilidades en el proceso de aprendizaje de la química. Además, manifestaron que la nota asignada por sus compañeros fue justa y transparente. Aparece nuevamente la rúbrica como la herramienta que hace que la evaluación, y como consecuencia de esta, la nota, deje de ser relativa a los criterios de cada profesor.

\section{Agradecimiento}

Los autores expresan sus más sinceros agradecimientos a los estudiantes y profesores de la Universidad EIA por el apoyo durante la implementación de la estrategia metodológica.

\section{Referencias}

[1] P. Araque, "Propuesta de estrategia metodológica para el desarrollo de competencias en la elaboración y solución de problemas de química para estudiantes de primer semestre de ingeniería" KnE Engineering. 2018, pp. $205-215$.

[2] P. Araque, P. Diseño instruccional para el curso de Química General e Inorgánica de la Universidad EIA modalidad presencial orientada a la virtualidad. Universidad EIA, Proyecto de investigación, Universidad EIA 2017. Envigado-Colombia.

[3] P. Carlino, Enseñar a escribir en la universidad: ¿cómo lo hacen en Estados Unidos y por qué? Revista Iberoamericana de Educación., vol 2. (2), pp. 5767. 2002.

[4] C. Márquez, "Aprender ciencias a través del lenguaje". Revista Educar: revista de educación, vol. 33, pp. 27-38, 2005. 
[5] E. J. Picon, "La rúbrica y la justicia en la evaluación". Íkala, revista de lenguaje y cultura vol. 3,18, pp. 79-94. 2013.

[6] M. E. Cano, "La evaluación por competencias en la educación superior. Profesorado". Revista de currículum y formación del profesorado, vol. 3, 12, pp. 1-16. 2008.

[7] L. F. Bachman, A. S. Palmer, Language test development, Language testing in practice. 1 ed. Nueva York, Oxford University Press 1996, pp. 83-133.

[8] I. Mansoor, S. Grant, A, "writing rubric to assess ESL student performance". Adventures in Assessment, vol. 14, pp. 33-38, 2002. (ERIC Document Reproduction Service No. ED 482885).

[9] J.J. Torres, V.H. Perera, "La rúbrica como instrumento pedagógico para la tutorización y evaluación de los aprendizajes en el foro online en Educación Superior". Pixel-Bit. Revista de Medios y Educación, vol. 36, pp. 141-149. 2010.
[10] J. Williams, Preparing to teach writing: Research, theory and practice. 3 ed. Mahwah, Lawrence Erlbaum Associates., pp. 297-331. 2003.

[11] C. Gipps, "Socio-cultural aspects of assessment". Review of Research in Education, 24, pp. 355-392. 1999

[12] Tecnológico de Monterrey, Juego de Roles. Investigación e Innovación educativa Centro Virtual de Técnicas Didácticas. 2010. Tomado de: http://sitios.itesm.mx/va/dide2/tecnicas_didacticas/juego_roles.htm (25 de mayo de 2018).

[13] S. Beer, "Beyond Dispute - The Invention of Team Syntegrity", John Wiley \& Sons, Inc. pp. 380. 1994. ISBN: 978-0-471-94451-5.

[14] J. Jorba, N. Sanmartí. Enseñar, aprender y evaluar: un proceso de regulación continua. Propuestas didácticas para las áreas de ciencias de la naturaleza y matemáticas. Ministerio de Educación de España. España, pp. 18. 1996. 